\title{
The Last will be First, and the First Last: Segregation in Societies with Positional Externalities
}

Citation for published version (APA):

Herings, P. J-J., Saulle, R., \& Seel, C. (2018). The Last will be First, and the First Last: Segregation in Societies with Positional Externalities. Maastricht University, Graduate School of Business and

Economics. GSBE Research Memoranda No. 027 https://doi.org/10.26481/umagsb.2018027

Document status and date:

Published: 17/12/2018

DOI:

10.26481/umagsb.2018027

Document Version:

Publisher's PDF, also known as Version of record

\section{Please check the document version of this publication:}

- A submitted manuscript is the version of the article upon submission and before peer-review. There can be important differences between the submitted version and the official published version of record.

People interested in the research are advised to contact the author for the final version of the publication, or visit the DOI to the publisher's website.

- The final author version and the galley proof are versions of the publication after peer review.

- The final published version features the final layout of the paper including the volume, issue and page numbers.

Link to publication

\footnotetext{
General rights rights.

- You may freely distribute the URL identifying the publication in the public portal. please follow below link for the End User Agreement:

www.umlib.nl/taverne-license

Take down policy

If you believe that this document breaches copyright please contact us at:

repository@maastrichtuniversity.nl

providing details and we will investigate your claim.
}

Copyright and moral rights for the publications made accessible in the public portal are retained by the authors and/or other copyright owners and it is a condition of accessing publications that users recognise and abide by the legal requirements associated with these

- Users may download and print one copy of any publication from the public portal for the purpose of private study or research.

- You may not further distribute the material or use it for any profit-making activity or commercial gain

If the publication is distributed under the terms of Article $25 \mathrm{fa}$ of the Dutch Copyright Act, indicated by the "Taverne" license above, 


\title{
Maastricht University
}

\author{
P.J.J. Herings, R.D. Saulle, \\ C. Seel
}

The Last will be First, and the First Last: Segregation in Societies with Positional Externalities

RM/18/027

\section{GSBE}

Maastricht University School of Business and Economics

Graduate School of Business and Economics

P.O Box 616

NL- 6200 MD Maastricht

The Netherlands 


\title{
The Last will be First, and the First Last: Segregation in Societies with Positional Externalities
}

\author{
P. Jean-Jacques Herings ${ }^{*} \quad$ Riccardo D. Saulle ${ }^{\dagger} \quad$ Christian Seel ${ }^{\ddagger}$
}

December 12, 2018

\begin{abstract}
This paper studies coalition formation among individuals who differ in productivity. The output of a coalition is determined by the sum of productivities if the coalition exceeds a minimal threshold of members. We consider competitive societies in which the surplus of a coalition is split according to productivity and egalitarian societies in which coalitions split their surplus equally. Preferences of coalition members depend on their material payoffs, but are also influenced by positional concerns, which relate their material payoffs to the average material payoff in the coalition. Our analysis uses two stability notions, the Core and the Myopic Stable Set.

Both competitive and egalitarian societies lead to segregated partition structures. For competitive societies, all stable allocations are based on bottom-up segregation, i.e., individuals with adjacent productivities form coalitions and if some individuals are not part of a productive coalition, then these are the most productive ones. For egalitarian societies, we obtain top-down segregation in all stable allocations. Again it holds that individuals with adjacent productivities form coalitions, but now the least productive individuals may not be part of any productive coalition.

If all individuals have different productivity levels, then the material efficiency of competitive societies is below that of egalitarian societies.
\end{abstract}

KEYWORDS: Group Formation, Segregation, Relative Payoff, Egalitarianism, Meritocracy, Social Environment.

JEL classification: C70,C71,D62.

\footnotetext{
*Department of Economics, Maastricht University, Maastricht, The Netherlands. E-Mail: P.Herings@maastrichtuniversity.nl

${ }^{\dagger}$ Department of Economics, Maastricht University, Maastricht, The Netherlands. E-Mail:

R.Saulle@maastrichtuniversity.nl

${ }_{\ddagger}^{\ddagger}$ Department of Economics, Maastricht University, Maastricht, The Netherlands. E-Mail: C.Seel@maastrichtuniversity.nl
} 


\section{Introduction}

Coalition formation is an important pattern of human behavior and many social phenomena can be studied in terms of a coalition formation process. For example, think of the provision of a local public good, the bargaining process underlying environmental agreements, political party formation, cartel formation, and racial integration. Typically, we think about the coalition formation process as a voluntary one. And indeed, in coalitional games, we mostly assume that individuals cannot exert their power or abilities in order to subjugate others. ${ }^{1}$

Historically, the exercise of power has been pervasive to every society albeit being present in different forms. Those forms of coercion can be naturally traced back to the idea of the "state of nature", a hypothetical primitive scenario in which neither property rights nor sociopolitical institutions of enforcement exist. According to the political philosopher Thomas Hobbes (1651), this condition of "mere nature" induces a bellum omnium contra omnes in which the most advantaged individuals are the physically strongest ones.

In the state of nature, however, individuals share the wish of ending the conflicts in order to claim properties and to benefit from their goods. This common desire leads into a "social contract" which bans the coercive force in favor of the enforcement power of institutions which ensure social norms and property rights. ${ }^{2}$ In broad economic terms, different societies can be seen as the outcome of different social contracts.

In this paper, we model two possible and alternative outcomes of a social contract, a competitive and an egalitarian society. The competitive society is characterized by the fact that it does not allow coercion, but there is no enforcement of resource redistribution within it, i.e., each member of a coalition receives a material payoff which reflects the member's ability. The egalitarian society also does not allow for coercive force, but guarantees through institutions or norms an equal distribution of resources within a coalition, i.e., each member of a coalition receives the same material payoff.

In addition to the material payoff, in our model, preferences depend on a notion of positional concern. The phenomenon of positional concern, especially about income and consumption, was first elaborated by Duesenberry (1949) who proposed the "demonstration effect" to explain how a family's consumption is influenced by the purchases of its neighbors. Based on this observation, he developed the "relative income hypothesis" which states that the level of consumption satisfaction of an individual depends on his relative rank in the society.

\footnotetext{
${ }^{1}$ Notable exceptions of models with different forms of coercion are due to Piccione and Rubinstein (2007), Acemoglu, Egorov and Sonin (2008), and Piccione and Razin (2009).

${ }^{2}$ On this point of view, see Muthoo (2004) and Hafer (2006) who study models explaining the rise of property rights from the state of nature.
} 
Following the same line of reasoning, Frank (1985) argues that positional concerns can explain many real world phenomena such as flatter intrafirm wage profiles in which low productivity workers and high productivity workers are paid more and less with respect to their marginal contribution to compensate for the difference in relative ranking. More recently, Card, Mas, Moretti, and Saez (2012) have shown that high relative wages affect job satisfaction and Bracha, Gneezy, and Loewenstein (2015) have shown a positive correlation between the supply of labor and relative wages. Moreover, happiness is found to be significantly and negatively affected by a lower relative income (Ferrer-i-Carbonell, 2005; Luttmer, 2005; Clark, Frijters, and Shields, 2008). To sum up, it is widely accepted that people do not only care about their absolute wealth, but also about their relative ranking in society.

Motivated by these findings, we propose a coalition formation model with positional externalities. A state in this model corresponds to a particular partition of the individuals into coalitions. We model the importance of the relative position in a coalition by the intragroup relative payoff. This measures the difference between the material payoff of an individual and the average material payoff of the coalition to which the individual belongs.

For the analysis of competitive and egalitarian societies, we introduce two notions of segregation, bottom-up segregation and top-down segregation. Roughly speaking, a state is bottom-up segregated if every coalition is formed by individuals that are adjacent in abilities and only the most productive individuals may be not be part of any productive coalition. On the opposite, a state is top-down segregated if every coalition is formed by individuals with adjacent productivities, but only the weakest individuals may not be part of any productive coalition.

We employ two different solution concepts to analyze the different societies. First, the classic concept of the Core which consists of all allocations for which there is no coalition such that all members benefit from deviating. As a second solution concept, we consider the Myopic Stable Set as introduced in Demuynck, Herings, Saulle, and Seel (2018). In the setting of this paper, the Myopic Stable Set is the minimal set of states such that (i) from any coalition structure in the set, no sequence of myopic coalitional improvements leaves the set and (ii) for any given initial coalition structure, there is a sequence of myopic coalitional improvements which leads to an element in the set.

For both competitive and egalitarian societies, we provide a full characterization of the Core and the Myopic Stable Set and show that both solution concepts coincide. In particular, we prove that for competitive societies the Myopic Stable Set consists of the set of bottom-up segregated states and for egalitarian societies the Myopic Stable Set consists of the set of top-down segregated states. This finding might seem counterintuitive at first glance as one might expect that the most advantaged individuals are best-off in competitive societies and the least advantaged individuals are best-off in egalitarian societies. 
The reasoning behind the predictions for competitive societies is as follows. First, note that the individuals with the lowest productivity are very attractive as coalition members, since they yield a higher relative payoff to other members of the coalition. Since these incentives are mutual, groups of individuals with low productivity will form. Intuitively, a similar process repeats until only individuals with high productivity might remain in unproductive coalitions. By contrast, the results for egalitarian societies rely on the fact that in an egalitarian society there is no envy among individuals, i.e. the relative payoff of each individual in each coalition is the same. Thus, individuals prefer to stay with highly productive individuals in order to increase their material payoff.

We conclude by providing a comparative welfare analysis between the two types of societies. When all individuals differ in productivity, stable states in egalitarian societies are always at least as materially efficient as stable states in competitive societies. This is caused by the fact that in an egalitarian society, the most productive individuals are always part of a productive coalition, while this might not be the case for competitive societies. We show that in the general case, with arbitrary productivities, there can be stable states in a competitive society which lead to strictly higher material efficiency than all stable states in egalitarian societies.

\section{$1.1 \quad$ Related Literature}

This paper is part of the vast literature on coalition formation theory (e.g. Ray, 2007; Ray and Vohra, 2014) and it is in line with contributions on hedonic games (e.g. Banerjee, Konishi, and Sönmez, 2001; Bogomolnaia and Jackson, 2002), social status (Milchtaich and Winter, 2002), and segregation (e.g. Goyal, Hernández, Martínez-Cánovas, Moisan, MuñozHerrera, and Sánchez, 2018).

In particular, our work is closely related to an increasing branch of literature devoted to the role of social norms in group formation (e.g. Watts, 2007; Piccione and Razin, 2009; Barberà, Beviá, and Ponsatí, 2015; Morelli and Park, 2016; Beviá, Córchon, and RomeroMedina, 2017). As in Piccione and Razin (2009), Morelli and Park (2016), and Barberà, Beviá, and Ponsatí (2015), we consider a model of coalition formation in which agents are vertically differentiated in productivity.

Piccione and Razin (2009) study a partition function game in which the relative strength of coalitions is represented by an exogenous "power relation". Agents care only about their social ranking, which is determined by their own individual power and by the power of the coalition to which they belong.

Morelli and Park (2016) study how the degree of heterogeneity in the remuneration of the individuals affects the number of rival coalitions. The heterogeneity in remuneration is modeled by a ranking rule defined on the imputation rule. Moreover, they distinguish 
between productivity and material efficiency defining the last one as an increasing function with respect to the cardinality of the coalition. In contrast to Morelli and Park (2016), we define the ranking rule on productivity levels.

In line with Barberà, Beviá, and Ponsatí (2015), we consider a stylized model of a society consisting of individuals, a productivity vector, and a productivity threshold. We study two possible allocation rules, the egalitarian one and the competitive one. In contrast to Barberà, Beviá, and Ponsatí (2015), we incorporate positional concerns into our analysis.

Since we implement a ranking rule in the preference structure, our contribution can be also broadly related to the literature on social preferences (e.g. Watts, 2007; Dufwenberg, Heidhues, Kirchsteiger, Riedel, and Sobel, 2011; Maccheroni, Marinacci, and Rustichini, 2012). In particular, from this point of view, it is in line with Watts (2007), who studies two different settings. In one of them, individuals prefer coalitions with high-skilled members and in the other individuals prefer coalitions with low-skilled members. In Watts (2007), the number of coalitions is fixed and ties are broken in favor of the biggest coalition.

\section{$2 \quad$ Model and Key Definitions}

We first describe the building blocks of a society $(N, \lambda, \rho)$. The finite set $N=\{1, \ldots, n\}$ consists of all individuals in the society. A coalition $S$ is a non-empty subset of $N$ and the collection of all possible non-empty coalitions is denoted by $\mathcal{N}=2^{N} \backslash\{\varnothing\}$. The collection of all partitions of a coalition $S \in \mathcal{N}$ is denoted by $\mathbb{P}(S)$. The set $\mathbb{P}(N)$ is denoted by $X$ and is called the state space. An element $x \in X$ is called a state and we write $|x|$ for the number of coalitions in $x$.

Let $\lambda \in \mathbb{R}_{++}^{N}$ be a productivity vector, where $\lambda_{i}$ represents the productivity level of individual $i$. Without loss of generality, the individuals in $N$ are indexed in decreasing order of productivity, i.e., $\lambda_{1} \geqslant \lambda_{2} \geqslant \cdots \geqslant \lambda_{n}$. Let some coalition $S \in \mathcal{N}$ be given. An individual $i \in S$ such that $\lambda_{i} \leqslant \lambda_{j}\left(\lambda_{i} \geqslant \lambda_{j}\right)$ for all $j \in S$ is called a weak (strong) individual in $S$. Let $w(S)=\max S$ and $s(S)=\min S$ denote the highest and the lowest numbered individual in coalition $S$, respectively. Notice that $w(S)$ is a weak and $s(S)$ is a strong individual in $S$. For $S=N$, we drop the reference to the coalition and simply refer to an individual $i$ as a weak or a strong individual.

The productivity threshold $\rho \in \mathbb{N}$ denotes the minimal level below which a coalition is not productive. To make the problem interesting, we assume throughout the paper that $\rho \leqslant n$. The surplus $u(S)$ of coalition $S \in \mathcal{N}$ equals $u(S)=\sum_{i \in S} \lambda_{i}$ if $|S| \geqslant \rho$ and 0 otherwise. The average surplus in coalition $S$ is denoted by $\bar{u}(S)=u(S) /|S|$. To get an intuition for the surplus function, think of the coalition as a firm which produces using a production technology which requires a minimal number of workers to produce any output and exhibits 
constant returns to scale above this threshold. ${ }^{3}$

Our next step is to specify how a coalition $S \in \mathcal{N}$ distributes its surplus over the coalition members. We denote the material payoff of individual $i$ in coalition $S$ by $m_{i}(S) \in \mathbb{R}$. Members of unproductive coalitions have a material payoff equal to zero. For members of productive coalitions, we consider two natural ways to divide the surplus. A division is competitive if each member of a productive coalition is paid according to productivity. A division is egalitarian if each member of a productive coalition receives the same payoff. These restrictions could be legal requirements, but they can also be thought of as social norms within a society.

Definition 2.1 (Competitive Society). A society $(N, \lambda, \rho)$ is competitive if surplus division within coalitions is competitive, i.e., for all $S \in \mathcal{N}$ and for all $i \in S, m_{i}(S)=\lambda_{i}$ if $|S| \geqslant \rho$ and $m_{i}(S)=0$ otherwise.

Definition 2.2 (Egalitarian Society). A society $(N, \lambda, \rho)$ is egalitarian if surplus division within coalitions is egalitarian, i.e., for all $S \in \mathcal{N}$ and for all $i \in S, m_{i}(S)=\bar{u}(S)$ if $|S| \geqslant \rho$ and $m_{i}(S)=0$ otherwise.

Since it will be clear from the context which of the two types of societies we are studying, we do not reflect the type of society in the notation for the material payoffs. We incorporate positional concerns through the following notion of relative payoff.

Definition 2.3 (Relative Payoff). Let $(N, \lambda, \rho)$ be a society. The relative payoff of individual $i \in N$ in coalition $S \in \mathcal{N}$ equals $r_{i}(S)=m_{i}(S)-\bar{u}(S)$.

The relative payoff measures the material payoff of individual $i$ relative to the average payoff in the individual's coalition. Note that both for competitive and egalitarian societies, the partition structure uniquely determines the payoffs and thereby the relative payoffs. Note also that in an egalitarian society, the relative payoff is equal to zero for each individual.

For every $x \in X$ and $i \in N$, we denote by $m_{i}(x)$ the material payoff of individual $i$ in state $x$, i.e., $m_{i}(x)=m_{i}\left(S_{i}(x)\right)$, where $S_{i}(x)$ is the coalition to which individual $i$ belongs in state $x$. Similarly, we write $r_{i}(x)=r_{i}\left(S_{i}(x)\right)$ for the relative payoff of individual $i$ in state $x$.

We are now ready to define the preferences of individual $i \in N$ over the state space. For every $x, y \in X$, we define relative payoff preferences $\geq_{i}$ over $X$ by setting $x \geq_{i} y$ if and only if either $m_{i}(x)>m_{i}(y)$ or both $m_{i}(x)=m_{i}(y)$ and $r_{i}(x) \geqslant r_{i}(y)$. Thus, individual $i$ prefers the state with the higher material payoff and if two states deliver the same material payoff, the one with the higher relative payoff. We denote the asymmetric part of $\geq_{i}$ by $>_{i}$.

\footnotetext{
${ }^{3}$ An alternative specification would be to define the threshold in terms of a minimum sum of productivities. This would lead to similar results.
} 
The final building block of our social environment is an effectivity correspondence $E$. For each pair of states $x, y \in X$, the effectivity correspondence $E$ associates a collection of coalitions $E(x, y) \subseteq \mathcal{N}$ which can move from $x$ to $y$. If $E(x, y)=\varnothing$, then no coalition can move from $x$ to $y$. If $T \in E(x, y)$, we say that coalition $T$ can move from state $x$ to state $y$.

We distinguish three groups that are affected by a move of $T$ from $x$ to $y$ : the moving individuals $T$ themselves, the residual individuals, and the unaffected individuals. The moving individuals $T$ create a new coalition and leave their former coalition members, the residual individuals, behind. Formally, for every $x, y \in X$ and $T \in E(x, y)$, the residual individuals are given by

$$
R(x, T)=\left\{i \in N \backslash T \mid S_{i}(x) \cap T \neq \varnothing\right\} .
$$

The unaffected coalitions $\mathcal{U}$ are the coalitions that are not influenced by the activity of the moving individuals. Formally, we have

$$
\mathcal{U}(x, T)=\{S \in x \mid S \cap T=\varnothing\}
$$

The members of the coalitions in $\mathcal{U}(x, T)$ are called the unaffected individuals and are collected in the set $U(x, T)$.

The related literature entertains different assumptions regarding the residual individuals. We consider the $\delta$-model (see Hart and Kurz, 1983) which prescribes that the residual individuals stay together. ${ }^{4}$ We also assume that the deviating coalition does not have the power to influence the unaffected individuals. The effectivity correspondence is therefore defined as

$$
E(x, y)=\left\{T \in \mathcal{N} \mid T \in y, \mathcal{U}(x, T) \subseteq y, \forall i \in R(x, T), S_{i}(x) \backslash T \in y\right\}, \quad x, y \in X
$$

This completes the description of the effectivity correspondence and thereby of our social environment $\Gamma=\left(N, X, E,\left\{\geq_{i}\right\}_{i \in N}\right)$ induced by a society $(N, \lambda, \rho)$.

It follows from the definition that, for every $x \in X, E(x, x)=x$, that is, any coalition in $x$ can choose to stay at $x$. Proposition 2.4 shows that for every $x, y \in X$ such that $x \neq y$, at most two coalitions can be part of $E(x, y)$.

Proposition 2.4. Let $\Gamma=\left(N, X, E,\left\{\geq_{i}\right\}_{i \in N}\right)$ be a social environment induced by a society. For all $x, y \in X$ such that $x \neq y$, we have $|E(x, y)| \in\{0,1,2\}$.

Proof. We distinguish two cases: either the moving individuals were part of one coalition or they were part of two or more coalitions before moving.

\footnotetext{
${ }^{4}$ An alternative specification is the $\gamma$-model, which prescribes that residual individuals fall apart into singleton coalitions. This would lead to similar results.
} 
Case 1: For all $T \in y$, there exists $S \in x$ such that $T \subseteq S$.

If $E(x, y)=\varnothing$, we are done, so assume $E(x, y) \neq \varnothing$. Since $x \neq y$, there exists $T \in y$ and $S \in x$ such that $T \subsetneq S$. Because we are in Case 1 and $E(x, y) \neq \varnothing$, the coalition $S$ is uniquely determined. Observe that $T$ cannot be an unaffected coalition. Hence, $T$ can only be a moving coalition or a coalition consisting of residual individuals. In the latter case, $S \backslash T$ is equal to the moving coalition. It follows that $E(x, y)=\{T, S \backslash T\}$.

Case 2: There exists $T \in y$ such that, for all $S \in x$, we have $T \backslash S \neq \varnothing$.

If $E(x, y)=\varnothing$, we are done, so assume $E(x, y) \neq \varnothing$. Since $T$ cannot be an unaffected or a residual coalition, the only way to go from $x$ to $y$ is by a move of $T$. It follows that $E(x, y)=\{T\}$.

\section{Stability}

In what follows, we define two related notions, the Core and the Myopic Stable Set. Both rely on a particular dominance notion. To define it, we write $y(x, S) \in X$ to denote the state that results from $E$ when the current state is $x$ and the moving coalition is $S$.

Definition 3.1 (Strict Dominance). Let $\Gamma=\left(N, X, E,\left\{\geq_{i}\right\}_{i \in N}\right)$ be a social environment induced by a society. A state $y \in X$ strictly dominates $x \in X$ by $S \in \mathcal{N}$ if $y=y(x, S)$ and $y>_{i} x$ for all $i \in S$.

Let a coalition $S \in \mathcal{N}$ be given. Let $f_{S}: X \rightarrow X$ be the coalitional dominance correspondence, where, for every $x \in X, f_{S}(x)$ denotes the set of states that strictly dominate $x$ by $S$. Note that $f_{S}(x)$ is either the singleton $\{y(x, S)\}$ or the empty set. We write $f(x)=\bigcup_{S \in \mathcal{N}} f_{S}(x)$ to denote the subset of $X$ consisting of all states that strictly dominate $x$. Such states are also referred to as myopic improvements upon $x$. The Core equals the set of states which are not strictly dominated by another state.

Definition 3.2 (Core). Let $\Gamma=\left(N, X, E,\left\{\geq_{i}\right\}_{i \in N}\right)$ be a social environment induced by a society. The Core equals the set of states $C=\{x \in X \mid f(x)=\varnothing\}$.

To define the Myopic Stable Set, it is convenient to define $\tilde{f}(x)=f(x) \cup\{x\}$, so $\tilde{f}(x)$ is obtained from $f(x)$ by adding the state $x$. We define the two-fold composition of $\tilde{f}$ by

$$
\tilde{f}^{2}(x)=\{z \in X \mid \exists y \in \tilde{f}(x): z \in \tilde{f}(y)\} .
$$

For $k \in \mathbb{N}$, we define the $k$-fold iteration $\tilde{f}^{k}(x)$ by induction as

$$
\tilde{f}^{k}(x)=\left\{z \in X \mid \exists y \in \tilde{f}^{k-1}(x): z \in \tilde{f}(y)\right\} .
$$


We define the set of all states that can be reached from $x$ by a finite number of dominations by

$$
\tilde{f}^{\mathbb{N}}(x)=\bigcup_{k \in \mathbb{N}} \tilde{f}^{k}(x)
$$

Because the set $X$ is finite, there is $k^{\prime} \in \mathbb{N}$ such that, for every $k \geqslant k^{\prime}, \tilde{f}^{\mathbb{N}}(x)=\tilde{f}^{k}(x)$.

We are now ready to introduce the Myopic Stable Set, following the definition of Demuynck, Herings, Saulle, and Seel (2018) for general social environments. ${ }^{5}$

Definition 3.3 (Myopic Stable Set). Let $\Gamma=\left(N, X, E,\left\{\geq_{i}\right\}_{i \in N}\right)$ be a social environment induced by a society. The set $M \subseteq X$ is a Myopic Stable Set if it satisfies the following three conditions:

1. Deterrence of external deviations: For every state $x \in M, f(x) \subseteq M$.

2. Iterated external stability: For every $y \in X \backslash M$, we have that $\tilde{f}^{\mathbb{N}}(y) \cap M \neq \varnothing$.

3. Minimality: There is no set $M^{\prime} \subsetneq M$ that satisfies Conditions 1 and 2 .

The first condition requires that no state in the set is dominated by a state outside the set. By the second condition, from any state outside the set, there is a finite sequence of dominations which reaches the set. The final condition requires the set to be a minimal set which satisfies the first two conditions.

A Myopic Stable Set is based on a myopic notion of dominance as agents or coalitions do not predict how their decision to change the current state will lead to further changes by other agents or coalitions. Such a notion is natural in complex social environments where the number of possible states is large and agents have little information about the possible actions other agents may take or the incentives of other agents. As shown in Demuynck, Herings, Saulle, and Seel (2018), for finite state spaces there is an equivalence between the set of recurrent states of any dynamic process that selects all myopic improvements with positive probability and the Myopic Stable Set.

In the remainder of this section, we provide some auxiliary results which will be helpful in establishing the main results in the next section. The first set of results follow directly from previous work. More specifically, by Theorem 3.4 in Demuynck, Herings, Saulle, and Seel (2018), if the dominance correspondence $\tilde{f}$ is lower hemi-continuous then the Myopic Stable Set is unique. Notice that lower hemi-continuity is trivially satisfied for finite state spaces. Thus, the Myopic Stable Set of the social environment induced by a society is always

\footnotetext{
${ }^{5}$ Demuynck, Herings, Saulle, and Seel (2018) allow for social environments with an infinite state space. For the finite case, their closedness requirement is automatically satisfied and $\tilde{f}^{\infty}(y)$, the closure of $\tilde{f}^{\mathbb{N}}(y)$, can be replaced by $\tilde{f}^{\mathbb{N}}(y)$ in the definition of iterated external stability. Thus, for the finite case, the definition of Myopic Stable Set in Demuynck, Herings, Saulle, and Seel (2018) is equivalent to Definition 3.3.
} 
unique. The property of internal external stability guarantees that the Myopic Stable Set is non-empty. Moreover, by Corollary 3.11 in Demuynck, Herings, Saulle, and Seel (2018), the Core is a subset of the Myopic Stable Set. Contrary to the concept of the Core, which only requires that there is no deviation away from one of its elements, the property of iterated external stability guarantees that the Myopic Stable Set can be reached from any initial state.

We establish a simple but useful result for the case in which some individuals have the same productivity level. Intuitively, if a state in which these individuals are in different coalitions is in the Core, then all states in which these individuals swap their coalitions are also in the Core. To define this formally, for given $x, y \in X$, let $b: N \rightarrow N$ be a productivity preserving permutation, i.e., $b$ is a one-to-one mapping such that $b(i)=j$ implies $\lambda_{i}=\lambda_{j}$. Moreover, we define $b(S)=\{j \in N \mid \exists i \in S$ such that $j=b(i)\}$.

Definition 3.4 (Equivalence). Let $(N, \lambda, \rho)$ be a society. The states $x$ and $y$ in $X$ are equivalent if there is a productivity preserving permutation $b$ such that for all coalitions $S \in x$ it holds that $b(S) \in y$.

Since $b$ can be the identity, any state $x$ is equivalent to itself. We denote the equivalence class of a state $x$ by $[x]$. The next result shows if a state is in the core, than all equivalent states are also in the core.

Proposition 3.5. Let $\Gamma=\left(N, X, E,\left\{\geq_{i}\right\}_{i \in N}\right)$ be a social environment induced by a competitive or an egalitarian society. If $x \in C$, then $[x] \subseteq C$.

Proof. Assume $x \in C$ and $y \in[x]$. Let $b$ be a productivity preserving permutation such that for all coalitions $S \in x$ it holds that $b(S) \in y$. Suppose $y \notin C$. Then there exists $z \in X$ and $T \in \mathcal{N}$ such that $z \in f_{T}(y)$. We define $b^{-1}(z)=\{S \in \mathcal{N} \mid b(S) \in z\}$. It holds that $b^{-1}(z) \in f_{b^{-1}(T)}(x)$, contradicting that $x \in C$.

We do not establish the corresponding analogue of Proposition 3.5 for the Myopic Stable Set at this point, since it does not admit a simple proof. In the next section, however, we show that the predictions of the Myopic Stable Set and the Core coincide. Thus, if the Myopic Stable Set contains a state $x$, it also contains all states in $[x]$.

\section{Segregation}

In this section, we introduce different notions of segregation. In our framework, segregation means that coalitions are formed between individuals with adjacent productivity levels. We first define segregation at the coalitional level. 
Definition 4.1 (Segregated Coalition). Let $(N, \lambda, \rho)$ be a society. A coalition $S \in \mathcal{N}$ is segregated if for all $i, j \in S$ with $\lambda_{i}<\lambda_{j}$ there is no $k \in N \backslash S$ such that $\lambda_{i}<\lambda_{k}<\lambda_{j}$.

To facilitate a formal definition of segregation at the societal level, we denote the set of all unproductive individuals at state $x$ by $Z(x)=\left\{i \in N|| S_{i}(x) \mid<\rho\right\}$ and the collection of all productive coalitions at state $x$ by $\mathcal{P}(x)=\{S \in x|| S \mid \geqslant \rho\}$. We introduce two notions which capture the idea of segregation at the societal level. The first one is called bottomup segregation. A state is bottom-up segregated if it satisfies three properties: coalitional assortativity, homophily, and exclusion of the strong.

Definition 4.2 (Bottom-up Segregated State). Let $(N, \lambda, \rho)$ be a society. The state $x \in X$ is bottom-up segregated if the following three properties hold:

i. Coalitional assortativity: For every $S, T \in \mathcal{P}(x)$ with $S \neq T$, we have $\lambda_{w(S)} \geqslant \lambda_{s(T)}$ or $\lambda_{w(T)} \geqslant \lambda_{s(S)}$.

ii. Homophily: If $S \in x$ satisfies $|S|>\rho$, then, for all $i, j \in S$, we have $\lambda_{i}=\lambda_{j}$.

iii. Exclusion of the strong: It holds that $|Z(x)|<\rho$. If $Z(x) \neq \varnothing$, then for every $i \in Z(x)$ and for every $j \in N \backslash Z(x)$, we have $\lambda_{i} \geqslant \lambda_{j}$.

Coalitional assortativity requires that given any two productive coalitions, a weak individual in one coalition has weakly higher productivity than a strong individual in the other coalition. Homophily states that if a coalition strictly exceeds the productivity threshold, then all individuals in the coalition have the same productivity. Exclusion of the strong means that only the strongest individuals may not be part of a productive coalition.

We denote the set of all bottom-up segregated states by $\underline{X}$.

Example 4.3. Consider a society $(N, \lambda, \rho)$ with $N=\{1, \ldots, 7\}$, productivities given by $\lambda_{1}=\cdots=\lambda_{3}>\lambda_{4}=\cdots=\lambda_{7}$, and a productivity threshold $\rho=2$. The state $x=$ $\{\{1,2\},\{3\},\{4,5,6,7\}\}$ is an example of a bottom-up segregated state. Since all individuals in a coalition have the same productivity, coalitional assortativity and homophily are trivially satisfied. Exclusion of the strong holds since the only member of an unproductive coalition is the strong individual 3.

The following lemma shows that productive coalitions in a bottom-up segregated state are indeed segregated.

Lemma 4.4. Let $(N, \lambda, \rho)$ be a society. If $x \in \underline{X}$ and $S \in \mathcal{P}(x)$, then $S$ is segregated. 
Proof. Suppose not. Let $x \in \underline{X}, S \in \mathcal{P}(x), i, j \in S$, and $k \in N \backslash S$ be such that $\lambda_{i}<\lambda_{k}<\lambda_{j}$. Let $T \in x$ be such that $k \in T$. By homophily, it holds that $|S|=\rho$. If $T \in \mathcal{P}(x)$, then, by coalitional assortativity, $\lambda_{w(S)} \geqslant \lambda_{s(T)} \geqslant \lambda_{k}$ or $\lambda_{k} \geqslant \lambda_{w(T)} \geqslant \lambda_{s(S)}$, which contradicts $i, j \in S$ and $\lambda_{i}<\lambda_{k}<\lambda_{j}$. If $T \notin \mathcal{P}(x)$, then $k \in Z(x)$, so by exclusion of the strong we have $\lambda_{k} \geqslant \lambda_{s(S)}$, leading to a contradiction with $j \in S$ and $\lambda_{k}<\lambda_{j}$.

In what follows, we provide an algorithmic procedure to find all bottom-up segregated states of a given society. The procedure results in a weighted directed rooted tree $\left(V, A, v^{0}\right)$, where $V$ is a set of decision nodes, $A \subseteq V \times V$ is a set of ordered pairs of nodes called arcs, and $v^{0}$ is a given initial decision node. To each arc $a \in A$, we associate an integer $\mu_{a} \in \mathbb{N}$ called its weight. This weight $\mu_{a}$ corresponds to the size of the coalition that is formed at the current decision node. Given some $v \in V$, we denote by $A^{v} \subseteq A$ the set of all arcs on the directed path from $v^{0}$ to $v$. Since $\left(V, A, v^{0}\right)$ is a directed rooted tree, the set $A^{v}$ is uniquely determined. To each decision node $v$, we associate a number $n(v)$ corresponding to the number of individuals that are not yet assigned to a coalition.

Procedure 4.5. Let $(N, \lambda, \rho)$ be a society. Let $v=v^{0}$ with $v^{0}$ a given decision node. The initial set of $\operatorname{arcs} A$ is equal to the empty set.

Step 1 Define $n(v)=n-\sum_{a \in A^{v}} \mu_{a}$. If $n(v) \geqslant \rho$, then add

$$
k=\max \left\{1, n(v)-\min \left\{i \in\{1, \ldots, n(v)\} \mid \lambda_{i}=\lambda_{n(v)}\right\}-\rho+2\right\}
$$

arcs starting at $v$ and ending at distinct new decision nodes. The arcs all have different weights, ranging from $\rho$ to $\rho+k-1$. If $0 \leqslant n(v)<\rho$, then $v$ is a terminal decision node and no new decision node is created.

Step 2 For each new decision node created in Step 1, go back to Step 1. Step 2 finishes when there are no new decision nodes anymore.

Step 3 Consider the weighted rooted directed tree $\left(V, A, v^{0}\right)$ resulting from Steps 1 and 2. For every terminal node $v$, let $\left(a_{1}, \ldots, a_{\ell}\right)$ be the arcs on the path from $v^{0}$ to $v$, and, for every partition $P \in \mathbb{P}\left\{n-\sum_{k=1}^{\ell} \mu_{a_{k}}, \ldots, 1\right\}$, generate the state

$$
\left\{\left\{n, \ldots, n-\mu_{a_{1}}+1\right\}, \ldots,\left\{n-\sum_{k=1}^{\ell-1} \mu_{a_{k}}, \ldots, n-\sum_{k=1}^{\ell} \mu_{a_{k}}+1\right\}\right\} \cup P .
$$

Step 4 Collect all the states created in Step 3 and add all equivalent states.

Intuitively, Procedure 4.5 works as follows. First, the highest numbered individuals form a productive coalition that satisfies homogeneity. Then the remaining highest numbered individuals form the next productive coalition that satisfies homogeneity, and so on. If some 


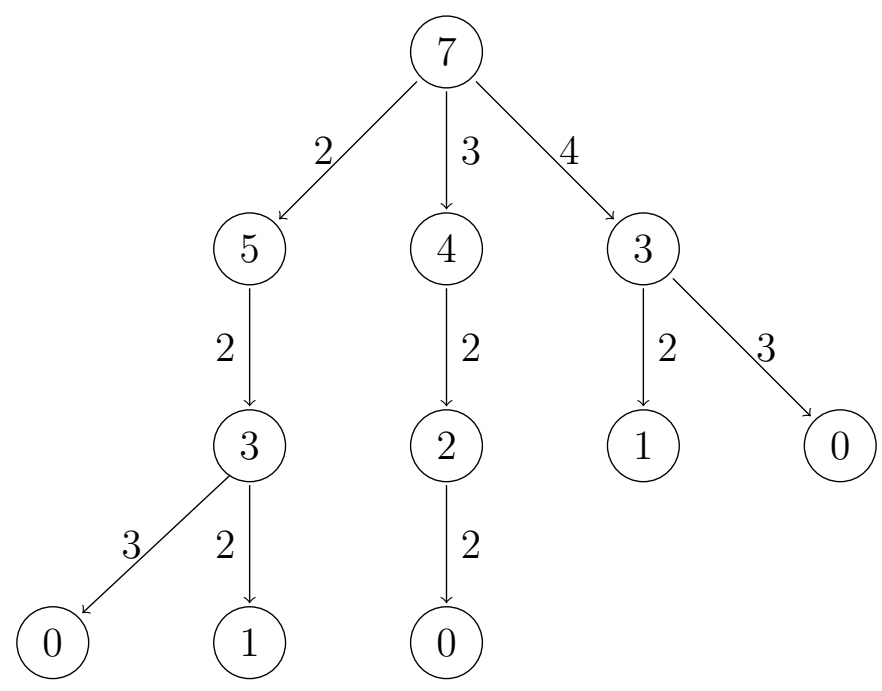

Figure 1: Result of Steps 1 and 2 of Procedure 4.5 for Example 4.3.

individuals are not part of a productive coalition of this bottom-up process, then those are the lowest numbered ones, i.e., the most productive ones, and they split up in an arbitrary way. The final step of Procedure 4.5 picks up equivalent states.

We use Example 4.3 to illustrate Procedure 4.5. The weighted directed rooted tree generated in Steps 1 and 2 is illustrated in Figure 1. This directed rooted tree has five terminal nodes, resulting in five states in Step 3:

$$
\begin{aligned}
& x_{1}=\{\{1,2,3\},\{4,5\},\{6,7\}\}, \\
& x_{2}=\{\{1\},\{2,3\},\{4,5\},\{6,7\}\}, \\
& x_{3}=\{\{1,2\},\{3,4\},\{5,6,7\}\}, \\
& x_{4}=\{\{1\},\{2,3\},\{4,5,6,7\}\}, \\
& x_{5}=\{\{1,2,3\},\{4,5,6,7\}\} .
\end{aligned}
$$

Step 4 adds equivalent states. By elementary combinatorics, this leads to two additional states for $x_{1}$, eight additional states for $x_{2}$, eleven additional states for $x_{3}$, two additional states for $x_{4}$, and no additional states for $x_{5}$. None of these states is identical. Thus, we have a total of 28 bottom-up segregated states. We refer to the appendix for a full enumeration of the bottom-up segregated states.

Proposition 4.6. Let $(N, \lambda, \rho)$ be a society. Procedure 4.5 generates the set of bottom-up segregated states $\underline{X}$.

Proof. See Appendix.

The second notion of societal segregation is top-down segregation. As bottom-up segregation, it requires three properties. The first two properties, coalitional assortativity and 
homophily, are identical as for bottom-down segregation. The third one replaces exclusion of the strong by exclusion of the weak, i.e., the least productive individuals can be excluded.

Definition 4.7 (Top-down Segregated State). Let $(N, \lambda, \rho)$ be a society. The state $x \in X$ is top-down segregated if the following three properties hold:

i. Coalitional assortativity: For every $S, T \in \mathcal{P}(x)$ with $S \neq T$, we have $\lambda_{w(S)} \geqslant \lambda_{s(T)}$ or $\lambda_{w(T)} \geqslant \lambda_{s(S)}$.

ii. Homophily: If $S \in x$ satisfies $|S|>\rho$, then, for all $i, j \in S$, we have $\lambda_{i}=\lambda_{j}$.

iii. Exclusion of the weak: It holds that $|Z(x)|<\rho$. If $Z(x) \neq \varnothing$, then for every $i \in Z(x)$ and for every $j \in N \backslash Z(x)$, we have $\lambda_{i} \leqslant \lambda_{j}$.

We denote the set of all top-down segregated states by $\bar{X}$. The following result states that every productive coalition in a top-down segregated state is segregated. The proof is analogous to Lemma 4.4 and therefore omitted.

Lemma 4.8. Let $(N, \lambda, \rho)$ be a society. If $x \in \bar{X}$ and $S \in \mathcal{P}(x)$, then $S$ is segregated.

The procedure to find all top-down segregated states also proceeds along the same lines as for bottom-down segregated states.

Procedure 4.9. Let $(N, \lambda, \rho)$ be a society. Let $v=v^{0}$ with $v^{0}$ a given decision node. The initial set of $\operatorname{arcs} A$ is equal to the empty set.

Step 1 Define $n(v)=n-\sum_{a \in A^{v}} \mu_{a}$. If $n(v) \geqslant \rho$, then add

$$
k=\max \left\{1, n(v)-\min \left\{i \in\{1, \ldots, n(v)\} \mid \lambda_{i}=\lambda_{n(v)}\right\}-\rho+2\right\}
$$

arcs starting at $v$ and ending at distinct new decision nodes. The arcs all have different weights, ranging from $\rho$ to $\rho+k-1$. If $0 \leqslant n(v)<\rho$, then $v$ is a terminal decision node and no new decision node is created.

Step 2 For each new decision node created in Step 1, go back to Step 1. Step 2 finishes when there are no new decision nodes anymore.

Step 3 Consider the weighted rooted directed tree $\left(V, A, v^{0}\right)$ resulting from Steps 1 and 2. For every terminal node $v$, let $\left(a_{1}, \ldots, a_{\ell}\right)$ be the arcs on the path from $v^{0}$ to $v$, and, for every partition $P \in \mathbb{P}\left\{\sum_{k=1}^{\ell} \mu_{a_{k}}+1, \ldots, n\right\}$ generate the state

$$
\left\{\left\{1, \ldots, \mu_{a_{1}}\right\}, \ldots,\left\{\sum_{k=1}^{\ell-1} \mu_{a_{k}}+1, \ldots, \sum_{k=1}^{\ell} \mu_{a_{k}}\right\}\right\} \cup P .
$$

Step 4 Collect all the states created in Step 3 and add all equivalent states. 
Proposition 4.10. Let $(N, \lambda, \rho)$ be a society. Procedure 4.9 generates the set of top-down segregated states $\bar{X}$.

As the procedure is the mirror image of the case for bottom-up segregation, we omit the proof that Procedure 4.9 results in the set of top-down segregated states.

\section{$5 \quad$ Results for Competitive and Egalitarian Societies}

In this section, we study the general properties of competitive and egalitarian societies with relative payoff preferences.

The following result is the main characterization result for competitive societies.

Theorem 5.1. Let $\Gamma=\left(N, X, E,\left\{\geq_{i}\right\}_{i \in N}\right)$ be the social environment induced by a competitive society $(N, \lambda, \rho)$. A state is in the Core of $\Gamma$ if and only if it is a bottom-up segregated state. Moreover, the Core coincides with the Myopic Stable Set.

Proof. See Appendix.

Both the Core and the Myopic Stable Set predict the set of bottom-up segregated states. Let us start with an intuition for the Core. An existing productive coalition has two different ways of improving its payoffs. Either it can get rid off a member with above average productivity or it can add less productive individuals to the coalition. When a state satisfies homophily, the first option does not work as any resulting coalition would be unproductive. The second option is prevented when a state satisfies coalitional assortativity as the less productive individuals would not want to switch to a more productive coalition. Finally, if the total number of individuals in unproductive coalitions is below $\rho$, an unproductive individual can only form coalitions with already productive individuals. However, if the unproductive individuals have a high productivity as a consequence of exclusion of the strong, other individuals will not form coalitions with them due to the resulting lower social rank.

The equivalence of the Core and the Myopic Stable Set gives an additional dynamic foundation for the prediction: from any state that is not bottom-up segregated, there is a path of myopic improvements to a bottom-up segregated state. In fact, any dynamic process that selects myopic improvements with positive probability will have the Myopic Stable Set as the set of recurrent states.

We now turn to the special case where all individuals differ in productivity.

Definition 5.2 (Complete Heterogeneity). A society $(N, \lambda, \rho)$ is completely heterogeneous if all individuals differ in their productivity, i.e., for all $i, j \in N$ with $i \neq j$, we have $\lambda_{i} \neq \lambda_{j}$. 
Let $\ell$ and $o$ be non-negative integers such that $o<\rho$ and the number of individuals in the society equals $n=\ell \rho+o$. The number $\ell$ is equal to the highest possible number of productive coalitions. For $k \in\{1, \ldots, \ell\}$, we define $B^{k}=\{n-\rho k+1, \ldots, n-(k-1) \rho\}$ and call it the $k^{\text {th }}$ bottom-up segregated coalition. These coalitions are collected in the set $\mathcal{B}=\left\{B^{k} \mid k=1, \ldots, \ell\right\}$.

Proposition 5.3. Let $\Gamma=\left(N, X, E,\left\{\geq_{i}\right\}_{i \in N}\right)$ be the social environment induced by the completely heterogeneous competitive society $(N, \lambda, \rho)$. Then the Core consists of those states which contain the collection $\mathcal{B}$, i.e., $C=\{x \in X \mid \mathcal{B} \subseteq x\}$.

Proof. By Theorem 5.1, the Core coincides with the set of bottom-up segregated states $\underline{X}$. By Proposition 4.6, Procedure 4.5 generates the entire set $\underline{X}$. Using the fact that the society is completely heterogeneous, it follows that in Step 1 of the procedure only arcs with weight $\rho$ are generated, leading to exactly the states in $\{x \in X \mid \mathcal{B} \subseteq x\}$ in Step 3. By the fact that the society is completely heterogeneous, there are no equivalent states $x$ and $y$ such that $x \neq y$. Therefore, no new states are added in Step 4 of Procedure 4.5.

The previous proposition uniquely characterizes the productive coalitions in the Core. It allows for an arbitrary partition of the unproductive individuals. Their material payoff is zero in either case and thus their relative payoff is also zero.

If $n$ is an integer multiple of $\rho$, then the Core is unique and it coincides with the collection of bottom-up segregated coalitions $\mathcal{B}$. Similarly, if $n=\rho \ell+1$, then the Core is unique and it coincides with the collection of bottom-up segregated coalitions $\mathcal{B}$ and the singleton $\{1\}$.

We now turn to the analysis of egalitarian societies. Recall that relative payoffs are now zero for all individuals. Thus, the preferences coincide with the preferences over the material payoffs. The following proposition is the main characterization result for egalitarian societies.

Theorem 5.4. Let $\Gamma=\left(N, X, E,\left\{\geq_{i}\right\}_{i \in N}\right)$ be the social environment induced by an egalitarian society $(N, \lambda, \rho)$. A state is in the Core of $\Gamma$ if and only if it is a top-down segregated state. Moreover, the Core coincides with the Myopic Stable Set.

Proof. See Appendix.

Contrary to the case of competitive societies, in egalitarian societies members of productive coalitions prefer to have more productive individuals in their coalition.

Let $\ell$ and $o$ be non-negative integers such that $o<\rho$ and the number of individuals in the society equals $n=\ell \rho+o$. For $k \in\{1, \ldots, \ell\}$, we define $T^{k}=\{\rho k-\rho+1, \ldots, \rho k\}$ and call it the $k^{\text {th }}$ top-down segregated coalition. These coalitions are collected in the set $\mathcal{T}=\left\{T^{k} \mid k=1, \ldots, \ell\right\}$. 
Proposition 5.5. Let $\Gamma=\left(N, X, E,\left\{\geq_{i}\right\}_{i \in N}\right)$ be the social environment induced by the completely heterogeneous egalitarian society $(N, \lambda, \rho)$. Then the Core consists of those states which contain the collection $\mathcal{T}$, i.e., $C=\{x \in X \mid \mathcal{T} \subseteq x\}$.

Proof. By Theorem 5.4, the Core coincides with the set of top-down segregated states $\bar{X}$. Procedure 4.9 generates the entire set $\bar{X}$. Using the fact that the society is completely heterogeneous, it follows that in Step 1 of the procedure only arcs with weight $\rho$ are generated, leading to exactly the states in $\{x \in X \mid \mathcal{T} \subseteq x\}$ in Step 3. By the fact that the society is completely heterogeneous, there are no equivalent states $x$ and $y$ such that $x \neq y$. Therefore, no new states are added in Step 4 of Procedure 4.9.

\section{Material Efficiency}

In this section, we compare competitive and egalitarian allocations in terms of the resulting material efficiency.

Definition 6.1 (Material Efficiency). Let $(N, \lambda, \rho)$ be a society. The material efficiency of a state $x \in X$ is equal to $\sum_{i \in N} m_{i}(x)$.

The maximal material efficiency a state $x \in X$ can reach is given by $\sum_{i \in N} \lambda_{i}$. We now compare competitive and egalitarian societies in terms of the resulting material efficiency.

Theorem 6.2. Let $(N, \lambda, \rho)$ be a completely heterogeneous society. Every Core element of the social environment induced by the egalitarian society is at least as materially efficient as every Core element of the social environment induced by the competitive society. They are equally materially efficient if and only if $n$ is an integer multiple of $\rho$.

Proof. Recall that in the competitive case the Core is equal to $\underline{X}=\{x \in X \mid \mathcal{B} \subseteq x\}$ by Proposition 5.3 and in the egalitarian case it equals $\bar{X}=\{x \in X \mid \mathcal{T} \subseteq x\}$ by Proposition 5.5.

Let some $\underline{x} \in \underline{X}$ and some $\bar{x} \in \bar{X}$ be given. We have that $\sum_{i \in N} m_{i}(\underline{x})=\sum_{i \in N} \lambda_{i}-\sum_{i \in Z(\underline{x})} \lambda_{i}$ and $\sum_{i \in N} m_{i}(\bar{x})=\sum_{i \in N} \lambda_{i}-\sum_{i \in Z(\bar{x})} \lambda_{i}$.

Let $\ell$ and $o$ be non-negative integers such that $o<\rho$ and the number of individuals in the society equals $n=\ell \rho+o$. As the society is completely heterogeneous, it holds that $\sum_{i \in Z(\underline{x})} \lambda_{i}=\left(\lambda_{1}+\cdots+\lambda_{o}\right) \geqslant\left(\lambda_{n-o+1}+\cdots+\lambda_{n}\right)=\sum_{i \in Z(\bar{x})} \lambda_{i}$, so $\sum_{i \in N} m_{i}(\underline{x}) \leqslant \sum_{i \in N} m_{i}(\bar{x})$, where equality holds if and only if $o=0$.

\section{Concluding Remarks}

We have studied coalition formation among individuals who differ in productivity. The joint output of a coalition is determined by the sum of productivities if a coalition exceeds a 
minimal threshold of members. We consider two types of societies, competitive societies in which the surplus of a coalition is split according to productivity and egalitarian societies in which coalitions split their surplus equally. The preferences of coalition members depend on the share of the surplus, but also on the relative payoff in the group.

Our analysis of the societies uses two different stability concepts, the classic static concept of the Core and the Myopic Stable Set, which enables a dynamic interpretation. The two concepts are shown to yield the same predictions.

Both competitive and egalitarian societies lead to segregated societies, i.e., coalitions are only formed between individuals with similar productivities. In contrast to a superficial intuition, the only individuals who might not be part of a productive coalition are the most productive individuals in a competitive society and the least productive individuals in an egalitarian society. To understand the intuition for these predictions, note that members of a competitive society will lose in social rank when forming coalitions with very productive individuals. Thus, the last (least productive individuals) are first in forming coalitions and the first (most productive individuals) last. The most productive individuals might even fail to attract other coalition members at all. On the contrary, in the egalitarian society the most productive individuals are very attractive as coalition members, since other coalition members gain from their productivity. 


\section{Appendix}

\section{A.1 Bottom-up Segregated States in the Example 4.3.}

\begin{tabular}{|c|l|}
\hline$\left[x_{1}\right]$ & $\{\{1,2,3\},\{4,5\},\{6,7\}\}$ \\
& $\{\{1,2,3\},\{4,6\},\{5,7\}\}$ \\
& $\{\{1,2,3\},\{4,7\},\{5,6\}\}$ \\
\hline$\left[x_{2}\right]$ & $\{\{1\},\{2,3\},\{4,5\},\{6,7\}\}$ \\
& $\{\{1\},\{2,3\},\{4,6\},\{5,7\}\}$ \\
& $\{\{1\},\{2,3\},\{4,7\},\{5,6\}\}$ \\
& $\{\{2\},\{1,3\},\{4,5\},\{6,7\}\}$ \\
& $\{\{2\},\{1,3\},\{4,6\},\{5,7\}\}$ \\
& $\{\{2\},\{1,3\},\{4,7\},\{5,6\}\}$ \\
& $\{\{3\},\{1,2\},\{4,5\},\{6,7\}\}$ \\
& $\{\{3\},\{1,2\},\{4,6\},\{5,7\}\}$ \\
& $\{\{3\},\{1,2\},\{4,7\},\{5,6\}\}$ \\
\hline$\left[x_{3}\right]$ & $\{\{1,2\},\{3,4\},\{5,6,7\}\}$ \\
& $\{\{1,2\},\{3,5\},\{4,6,7\}\}$ \\
& $\{\{1,2\},\{3,6\},\{4,5,7\}\}$ \\
& $\{\{1,2\},\{3,7\},\{4,5,6\}\}$ \\
& $\{\{1,3\},\{2,4\},\{5,6,7\}\}$ \\
& $\{\{1,3\},\{2,5\},\{4,6,7\}\}$ \\
& $\{\{1,3\},\{2,6\},\{4,5,7\}\}$ \\
& $\{\{1,3\},\{2,7\},\{4,5,6\}\}$ \\
& $\{\{2,3\},\{1,4\},\{5,6,7\}\}$ \\
& $\{\{2,3\},\{1,5\},\{4,6,7\}\}$ \\
& $\{\{2,3\},\{1,6\},\{4,5,7\}\}$ \\
& $\{\{2,3\},\{1,7\},\{4,5,6\}\}$ \\
\hline$\left[x_{4}\right]$ & $\{\{1\},\{2,3\},\{4,5,6,7\}\}$ \\
& $\{\{2\},\{1,3\},\{4,5,6,7\}\}$ \\
& $\{\{3\},\{1,2\},\{4,5,6,7\}\}$ \\
\hline$\left[x_{5}\right]$ & $\{\{1,2,3\},\{4,5,6,7\}\}$ \\
\hline
\end{tabular}

\section{A.2 Proof of Theorem 4.6}

Proof. We first show that any state generated by Procedure 4.5 is bottom-up segregated.

We start with a state generated in Step 3 from an arc $\left(a_{1}, \ldots, a_{\ell}\right)$. Since all productive coalitions consist of individuals with adjacent subscripts and hence adjacent productivities, 
we have coalitional assortativity. If, for some $k \in\{1, \ldots, \ell\}, \mu_{a_{k}}>\rho$, then it follows from the selection of the number of added arcs in Step 1 that all individuals involved in the associated coalition have the same productivity, so homophily is satisfied. Finally, the individuals in the set $\left\{n-\sum_{k=1}^{\ell} \mu_{a_{k}}, \ldots, 1\right\}$ cannot form a productive coalition themselves and have weakly higher productivity than individuals in the productive coalitions, so exclusion of the strong is satisfied. To complete the first part of the proof, note that all three properties of a bottom-up segregated state are preserved under productivity preserving permutations, so the equivalent states generated in Step 4 are bottom-up segregated as well.

It remains to be shown that all bottom-up segregated states are generated by Procedure 4.5. To do so, we show that for each bottom-up segregated state, there is one permutation of a state generated in Steps 1-3. The key is to define the permutation/relabeling in a precise way.

Let $x=\left\{S^{1}, \ldots, S^{m}\right\} \in \underline{X}$ be a bottom-up segregated state with productive coalitions $\mathcal{P}(x)=\left\{S^{1}, \ldots, S^{\ell}\right\}$, where $S^{1}, \ldots, S^{\ell}$ are chosen such that

$$
\lambda_{s\left(S^{1}\right)} \leqslant \lambda_{w\left(S^{2}\right)} \leqslant \cdots \leqslant \lambda_{s\left(S^{\ell-1}\right)} \leqslant \lambda_{w\left(S^{\ell}\right)},
$$

and unproductive coalitions $S^{\ell+1}, \ldots, S^{m}$. For $k \in\{1, \ldots, m\}$, we denote the cardinality $\left|S^{k}\right|$ of coalition $S^{k}$ by $p_{k}$.

We define the permutation $b: N \rightarrow N$ as follows. Let some $i \in N$ be given. The number $k^{\prime} \in\{1, \ldots, \ell+1\}$ is uniquely determined in the following way. If

$$
i \in\left\{n-\sum_{k=1}^{\ell} p_{k}, \ldots, 1\right\}
$$

then $k^{\prime}=\ell+1$. Otherwise, $k^{\prime} \in\{1, \ldots, \ell\}$ is chosen such that

$$
i \in\left\{n-\sum_{k=1}^{k^{\prime}-1} p_{k}, \ldots, n-\sum_{k=1}^{k^{\prime}} p_{k}+1\right\} .
$$

In case $k^{\prime} \leqslant \ell$, we write $S^{k^{\prime}}=\left\{j_{1}, \ldots, j_{p_{k^{\prime}}}\right\}$ with $j_{1}>\cdots>j_{p_{k^{\prime}}}$ and define

$$
b(i)=j_{n-\sum_{k=1}^{k^{\prime}-1} p_{k}-i+1} .
$$

In case $k^{\prime}=\ell+1$, we write $S^{\ell+1} \cup \cdots \cup S^{m}=\left\{j_{1}, \ldots, j_{n-\sum_{k=1}^{\ell} p_{k}}\right\}$ with $j_{1}>\cdots>j_{n-\sum_{k=1}^{\ell} p_{k}}$ and define

$$
b(i)=j_{n-\sum_{k=1}^{\ell} p_{k}-i+1} .
$$

One of the directed paths $\left(a_{1}, \ldots, a_{\ell}\right)$ generated by Procedure 4.5 is such that, for $k=$ $1, \ldots, \ell, \mu_{a_{k}}=p_{k}$. Let the permutation $P \in \mathbb{P}\left\{n-\sum_{k=1}^{\ell} \mu_{a_{k}}, \ldots, 1\right\}$ be defined by $P=$ $\left\{b^{-1}\left(S^{k}\right) \mid k \in\{\ell+1, \ldots, m\}\right\}$. In Step 3, the directed path $\left(a_{1}, \ldots, a_{\ell}\right)$ together with the permutation $P$ generates the state

$$
y=\left\{\left\{n, \ldots, n-\mu_{a_{1}}+1\right\}, \ldots,\left\{n-\sum_{k=1}^{\ell-1} \mu_{a_{k}}, \ldots, n-\sum_{k=1}^{\ell} \mu_{a_{k}}+1\right\}\right\} \cup P .
$$


Clearly, for all $S \in y$ it holds that $b(S) \in x$. It remains to be shown that $b$ is productivity preserving, so $b(i)=j$ implies $\lambda_{i}=\lambda_{j}$, in order to conclude that $x \in[y]$, so is generated in Step 4 .

Suppose there is $i, j \in N$ such that $b(i)=j$ and $\lambda_{i} \neq \lambda_{j}$. Let $i^{\prime}$ be the highest numbered individual with this property and define $j^{\prime}=b\left(i^{\prime}\right)$. Since $x$ satisfies exclusion of the strong and by the construction of $b$, it cannot hold that $j^{\prime} \in S^{\ell+1} \cup \cdots \cup S^{m}$. Let $k^{\prime} \leqslant \ell$ be such that $j^{\prime} \in S^{k^{\prime}}$. Since $i^{\prime}$ is the highest numbered individual among $i \in N$ such that $\lambda_{i} \neq \lambda_{b(i)}$, it must hold that $\lambda_{i^{\prime}}<\lambda_{j^{\prime}}$. It follows that there is $j>i^{\prime}$ such that $j \notin S^{1} \cup \cdots \cup S^{k^{\prime}}$. Suppose there is $k \in\left\{k^{\prime}+1, \ldots, \ell\right\}$ such that $j \in S^{k}$. Since $\lambda_{w\left(S^{k}\right)} \leqslant \lambda_{j}<\lambda_{j^{\prime}} \leqslant \lambda_{s\left(S^{k^{\prime}}\right)}$, we obtain a contradiction to $x$ satisfying coalitional assortativity. Consequently, it holds that $j \in S^{\ell+1} \cup \cdots \cup S^{m}$. However, in that case $x$ violates exclusion of the strong since $\lambda_{j} \leqslant \lambda_{i^{\prime}}<\lambda_{j^{\prime}}$. We have obtained a contradiction. Consequently, $b(i)=j \operatorname{implies} \lambda_{i}=\lambda_{j}$.

\section{A.3 Proof of Theorem 5.1}

The following lemma is helpful in the proof of Theorem 5.1.

Lemma A.1. Let $\Gamma=\left(N, X, E,\left\{\geq_{i}\right\}_{i \in N}\right)$ be a social environment induced by a competitive society $(N, \lambda, \rho)$. Let $x \in X$ and $S \in x$ be such that $|S|>\rho$ and $\lambda_{i} \neq \lambda_{j}$ for some $i, j \in S$. Then $f_{S \backslash\{s(S)\}}(x) \neq \varnothing$.

Proof. Consider the coalition $T=S \backslash\{s(S)\}$. Since $|T| \geqslant \rho$, we have $m_{i}(T)=m_{i}(S)$ for all $i \in T$. Hence, to prove that $f_{S \backslash\{s(S)\}} \neq \varnothing$, it remains to show that $r_{i}(T)>r_{i}(S)$ for all $i \in T$.

Observe that $\lambda_{s(S)}>\bar{u}(S)$ since $\lambda_{i} \neq \lambda_{j}$ for some $i, j \in S$. Hence, for every $i \in T$, we have $\bar{u}(T)<\bar{u}(S)$ and thus $r_{i}(T)=m_{i}(T)-\bar{u}(T)>m_{i}(S)-\bar{u}(S)=r_{i}(S)$.

Proof of Theorem 5.1. The proof consists of three parts. We show first that $\underline{X} \subseteq C$, next

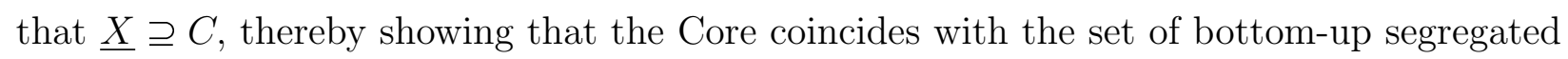
states, and finally that the Myopic Stable Set coincides with the Core.

Part 1. $\underline{X} \subseteq C$.

Let $\underline{x} \in X$ be a bottom-up segregated state, where $\underline{x}=\left\{S^{1}, \ldots, S^{m}\right\}, \mathcal{P}(\underline{x})=\left\{S^{1}, \ldots, S^{\ell}\right\}$, and, for every $k \in\{1, \ldots, \ell-1\}, \lambda_{s\left(S^{k}\right)} \leqslant \lambda_{w\left(S^{k+1}\right)}$. Moreover, we write $S^{1}=\left\{s_{1}^{1}, \ldots, s_{p}^{1}\right\}$ with $s_{1}^{1}<\cdots<s_{p}^{1}$.

Towards a contradiction, suppose that $\underline{x} \notin C$. Thus, there exists a coalition $T=$ $\left\{t_{1}, \ldots, t_{q}\right\}$ with $t_{1}<\cdots<t_{q}$ and a state $y$ such that $y \in f_{T}(\underline{x})$. Notice that any deviating coalition has to be productive, i.e., $|T| \geqslant \rho$. We proceed by induction.

Step 1: $S^{1} \cap T=\varnothing$. 
Notice that $S^{1}$ is productive. For all $i \in S^{1} \cap T$, it holds that $m_{i}\left(S^{1}\right)=m_{i}(T)$. Then a deviation has to rely on an improvement in relative payoff. We consider two cases: a case in which, for all $i, j \in S^{1}, \lambda_{i}=\lambda_{j}$ and a case in which there are some $i, j \in S^{1}$ with $\lambda_{i} \neq \lambda_{j}$.

In the former case, we have that $\bar{u}\left(S^{1}\right)=\lambda_{n} \leqslant \bar{u}(T)$ as, by assumption, $n$ is a weak individual in the society. Therefore, for all $i \in S^{1} \cap T$, it holds that

$$
r_{i}\left(S^{1}\right)=m_{i}\left(S^{1}\right)-\bar{u}\left(S^{1}\right) \geqslant m_{i}(T)-\bar{u}(T)=r_{i}(T)
$$

so it follows that $S^{1} \cap T=\varnothing$.

In the latter case, homophily of $\underline{x}$ implies that $\left|S^{1}\right|=\rho$. We define the collection of $\rho$ weakest individuals in $T$ by $T^{\prime}=\left\{t_{q-\rho+1}, \ldots, t_{q}\right\}$. It holds that $\bar{u}\left(T^{\prime}\right) \leqslant \bar{u}(T)$. For $i=0, \ldots, \rho-1$, it holds that $\lambda_{t_{q-i}} \geqslant \lambda_{s_{p-i}^{1}}$, so we have that $\bar{u}\left(S^{1}\right) \leqslant \bar{u}\left(T^{\prime}\right) \leqslant \bar{u}(T)$. Therefore, for every $i \in S^{1} \cap T$, it holds that

$$
r_{i}\left(S^{1}\right)=m_{i}\left(S^{1}\right)-\bar{u}\left(S^{1}\right) \geqslant m_{i}(T)-\bar{u}(T)=r_{i}(T),
$$

so it follows that $S^{1} \cap T=\varnothing$.

Step 2: If $\bigcup_{\kappa=1}^{k} S^{\kappa} \cap T=\varnothing$, then $S^{k+1} \cap T=\varnothing$.

If $k \geqslant \ell$, then $S^{k+1} \notin \mathcal{P}(\underline{x})$. As $\bigcup_{\kappa=1}^{k} S^{\kappa} \cap T=\varnothing$ and $|Z(\underline{x})|<\rho$ it follows that $|T|<\rho$, a contradiction to $|T| \geqslant \rho$.

If $k<\ell$, then $S^{k+1} \in \mathcal{P}(\underline{x})$ and we write $S^{k+1}=\left\{s_{1}^{k+1}, \ldots, s_{p}^{k+1}\right\}$ with $s_{1}^{k+1}<\ldots<s_{p}^{k+1}$. For every $i \in S^{k+1} \cap T$, it holds that $m_{i}\left(S^{k+1}\right)=m_{i}(T)$ since $|T| \geqslant \rho$. A deviation has to rely on an improvement in relative payoff. If for all $i, j \in S^{k+1}, \lambda_{i}=\lambda_{j}$, then $\bar{u}\left(S^{k+1}\right)=$ $\lambda_{s_{p}^{k+1}} \leqslant \bar{u}(T)$ as, by assumption, $s_{p}^{k+1}$ is a weak individual in $N \backslash \bigcup_{\kappa=1}^{k} S^{\kappa}$. Therefore, for all $i \in S^{k+1} \cap T$, it holds that

$$
r_{i}\left(S^{k+1}\right)=m_{i}\left(S^{k+1}\right)-\bar{u}\left(S^{k+1}\right) \geqslant m_{i}(T)-\bar{u}(T)=r_{i}(T),
$$

so $S^{k+1} \cap T=\varnothing$. If there is some $i, j \in S^{k+1}$ with $\lambda_{i} \neq \lambda_{j}$, by homophily, $\left|S^{k+1}\right|=\rho$. We define $T^{\prime}=\left\{t_{q-\rho+1}, \ldots, t_{q}\right\}$, so $\bar{u}\left(T^{\prime}\right) \leqslant \bar{u}(T)$. Since $\lambda_{t_{q-i}} \geqslant \lambda_{s_{p-i}^{k+1}}$ for $i=0, \ldots, \rho-1$, it follows that $\bar{u}\left(S^{k+1}\right) \leqslant \bar{u}\left(T^{\prime}\right) \leqslant \bar{u}(T)$. Therefore, for every $i \in S^{k+1} \cap T$, it holds that

$$
r_{i}\left(S^{k+1}\right)=m_{i}\left(S^{k+1}\right)-\bar{u}\left(S^{k+1}\right) \geqslant m_{i}(T)-\bar{u}(T)=r_{i}(T),
$$

so $S^{k+1} \cap T=\varnothing$.

By Steps 1 and 2 it follows that $T=\varnothing$ and we have obtained a contradiction. Consequently, it holds that $\underline{x} \in C$.

Part 2. $\underline{X} \supseteq C$. 
Let some $y \in C$ be given. Towards a contradiction, suppose that $y$ is not bottom-up segregated.

If homophily is violated, then there exists a coalition $S \in y$ with $|S|>\rho$ such that for some $i, j \in S, \lambda_{i}>\lambda_{j}$. Thus, by Lemma A.1, it holds that $f_{S \backslash\{s(S)\}}(y) \neq \varnothing$, a contradiction to $y$ being in the Core.

If coalitional assortativity is violated, then there are coalitions $S, T \in \mathcal{P}(y)$ such that $\lambda_{w(S)}<\lambda_{s(T)}$ and $\lambda_{w(T)}<\lambda_{s(S)}$. We write $S=\left\{s_{1}, \ldots, s_{p}\right\}$ and $T=\left\{t_{1}, \ldots, t_{q}\right\}$ with $s_{1}<\cdots<s_{p}$ and $t_{1}<\cdots<t_{q}$. We show that for $S^{\prime}=\left\{t_{q}, s_{2}, \ldots, s_{p}\right\}$ it holds that $f_{S^{\prime}}(y) \neq \varnothing$ or for $T^{\prime}=\left\{s_{p}, t_{2}, \ldots, t_{q}\right\}$ it holds that $f_{T^{\prime}}(y) \neq \varnothing$.

Since $S$ and $S^{\prime}$ are productive, we have $m_{i}(S)=m_{i}\left(S^{\prime}\right)$ for all $i \in S \cap S^{\prime}$. Moreover, since $|S|=\left|S^{\prime}\right|$ and $\lambda_{s_{1}}>\lambda_{t_{q}}$, we have

$$
\bar{u}(S)=\frac{\lambda_{s_{1}}+\sum_{i=2}^{p} \lambda_{s_{i}}}{|S|}>\frac{\lambda_{t_{q}}+\sum_{i=2}^{p} \lambda_{s_{i}}}{\left|S^{\prime}\right|}=\bar{u}\left(S^{\prime}\right) .
$$

Thus, for all $i \in S \cap S^{\prime}=S^{\prime} \backslash\left\{t_{q}\right\}$, it holds that $r_{i}(S)=m_{i}(S)-\bar{u}(S)<m_{i}\left(S^{\prime}\right)-\bar{u}\left(S^{\prime}\right)=$ $r_{i}\left(S^{\prime}\right)$. By symmetry, it also holds for all $i \in T \cap T^{\prime}=T^{\prime} \backslash\left\{s_{p}\right\}$ that $m_{i}(T)=m_{i}\left(T^{\prime}\right)$ and $r_{i}(T)<r_{i}\left(T^{\prime}\right)$.

Towards a contradiction, suppose that $s_{p}=w(S)$ has no incentive to participate in $T^{\prime}$ and $t_{q}=w(T)$ has no incentive to form $S^{\prime}$, so $r_{w(S)}(S) \geqslant r_{w(S)}\left(T^{\prime}\right)$ and $r_{w(T)}(T) \geqslant r_{w(T)}\left(S^{\prime}\right)$. Thus, by definition,

$$
\begin{aligned}
& m_{w(S)}(S)-\frac{\sum_{i \in S} \lambda_{i}}{p} \geqslant m_{w(S)}\left(T^{\prime}\right)-\frac{\lambda_{w(S)}}{q}-\frac{\sum_{i \in T \backslash\{s(T)\}} \lambda_{i}}{q}, \\
& m_{w(T)}(T)-\frac{\sum_{i \in T} \lambda_{i}}{q} \geqslant m_{w(T)}\left(S^{\prime}\right)-\frac{\lambda_{w(T)}}{p}-\frac{\sum_{i \in S \backslash\{s(S)\}} \lambda_{i}}{p} .
\end{aligned}
$$

These expressions can be simplified to get

$$
\begin{aligned}
p \lambda_{w(S)}+p \sum_{i \in T \backslash\{s(T)\}} \lambda_{i} \geqslant q \sum_{i \in S} \lambda_{i} \\
q \lambda_{w(T)}+q \sum_{i \in S \backslash\{s(S)\}} \lambda_{i} \geqslant p \sum_{i \in T} \lambda_{i} .
\end{aligned}
$$

Adding up these two inequalities and simplifying results in

$$
p \lambda_{w(S)}+q \lambda_{w(T)} \geqslant q \lambda_{s(S)}+p \lambda_{s(T)},
$$

leading to a contradiction since $\lambda_{w(S)}<\lambda_{s(T)}$ and $\lambda_{w(T)}<\lambda_{s(S)}$. Consequently, $s_{p}$ is strictly better off participating in $T^{\prime}$ or $t_{q}$ is strictly better off forming $S^{\prime}$.

We have shown that $f_{S^{\prime}}(y) \neq \varnothing$ or $f_{T^{\prime}}(y) \neq \varnothing$, contradicting that $y \in C$. Consequently, $y$ satisfies coalitional assortativity.

Suppose $y$ violates exclusion of the strong. If $|Z(y)| \geqslant \rho$, then we have $f_{Z(y)}(y) \neq \varnothing$, a contradiction. It remains to consider the case $|Z(y)|<\rho$. There exists $j \in Z(y), S \in \mathcal{P}(y)$, and $i \in S$ such that $\lambda_{j}<\lambda_{i}$. We write $S=\left\{s_{1}, \ldots, s_{p}\right\}$ with $s_{1}<\cdots<s_{p}$. Consider the 
coalition $T=\left\{j, s_{2}, \ldots, s_{p}\right\}$. We argue that $f_{T}(y) \neq \varnothing$. Notice that $m_{j}(Z(y))=0<m_{j}(T)$, so $j$ has an incentive to deviate. It remains to be shown that also members of $\left\{s_{2}, \ldots, s_{p}\right\}=$ $S \cap T$ have an incentive to deviate. For all $i \in S \cap T, m_{i}(S)=m_{i}(T)>0$. Since $|S|=|T|$, the following inequality holds,

$$
\bar{u}(S)=\frac{\lambda_{s_{1}}+\sum_{i=2}^{p} \lambda_{s_{i}}}{|S|}>\frac{\lambda_{j}+\sum_{i=2}^{p} \lambda_{s_{i}}}{|T|}=\bar{u}(T) .
$$

Therefore, for all $i \in S \cap T$, it holds that $r_{i}(S)=m_{i}(S)-\bar{u}(S)<m_{i}(T)-\bar{u}(T)=r_{i}(T)$. We have obtained a contradiction with $y \in C$. Consequently, $y$ satisfies exclusion of the strong.

\section{Part 3. The Myopic Stable Set coincides with the Core.}

Notice that the Core trivially satisfies deterrence of external deviations. In order to show that it coincides with the Myopic Stable Set, we have to show that it satisfies iterated external stability. The minimality requirement follows from the fact that the Core is a subset of the Myopic Stable Set.

The proof of iterated external stability is constructive using the following procedure, which, given an initial state $x_{0} \in X \backslash C$, generates a finite sequence of productive coalitions $S^{1}, \ldots, S^{\ell}$ and states $x_{1}, \ldots, x_{\ell}$ such that, for $k=1, \ldots, \ell, x_{k} \in \tilde{f}_{S^{k}}\left(x_{k-1}\right)$ and $x_{\ell}$ is a bottomup segregated state. The coalitions $S^{k}$ are all disjoint and chosen such that their members have the lowest productivity among players in $N \backslash\left(\cup_{\kappa=1}^{k-1} S^{\kappa}\right)$.

Start with a state $x_{0} \in X \backslash C$. Parts (i) and (ii) formalize a sequence of dominations where first a productive coalition with weak individuals forms and so forth until a strongest productive coalition forms. We define $k=1$.

(i) If $\left|N \backslash \bigcup_{\kappa=1}^{k-1} S^{\kappa}\right|<\rho$, then the procedure ends. Otherwise, move to (ii).

(ii) Consider a coalition $T \subseteq N \backslash \bigcup_{\kappa=1}^{k-1} S^{\kappa}$ with $|T|=\rho$ such that for all $i \in T$ and for all $j \in N \backslash\left(\bigcup_{\kappa=1}^{k-1} S^{\kappa} \cup T\right)$ it holds that $\lambda_{i} \leqslant \lambda_{j}$. If $f_{T}\left(x_{k-1}\right) \neq \varnothing$, then define $S^{k}=T$. If $f_{T}\left(x_{k-1}\right)=\varnothing$, then there is $i \in T$ who belongs to a productive coalition $S \in x_{k-1}$ such that $r_{i}(S) \geqslant r_{i}(T)$. For all $i \in S$, for all $j \in N \backslash\left(\bigcup_{\kappa=1}^{k-1} S^{\kappa} \cup S\right)$, it holds that $\lambda_{i} \leqslant \lambda_{j}$. In this case, define $S^{k}=S$. Define $x_{k}=y\left(x_{k-1}, S^{k}\right)$. Increase $k$ by 1 and move back to (i).

The procedure takes $\ell \leqslant n / \rho$ steps and finally generates the state $x_{\ell}$. We have that $\mathcal{P}\left(x_{\ell}\right)=\left\{S^{k} \mid k \in\{1, \ldots, \ell\}\right\}$. It holds that $x_{\ell} \in \tilde{f}^{\ell}\left(x_{0}\right)$ since, for every $k \in\{1, \ldots, \ell\}$, $x_{k} \in f_{S^{k}}\left(x_{k-1}\right)$ or $x_{k}=x_{k-1}$. It remains to be shown that $x_{\ell}$ belongs to the Core. By Parts 1 and 2 of the proof, we need to show that $x_{\ell}$ is bottom-up segregated.

We have that $\mathcal{P}\left(x_{\ell}\right)=\left\{S^{k} \mid k \in\{1, \ldots, \ell\}\right\}$. By construction it holds that $\lambda_{s\left(S^{1}\right)} \leqslant$ $\lambda_{w\left(S^{2}\right)} \leqslant \cdots \leqslant \lambda_{s\left(S^{\ell-1}\right)} \leqslant \lambda_{w\left(S^{\ell}\right)}$. It directly follows that for all $S, T \in \mathcal{P}\left(x_{\ell}\right)$ with $S \neq T$, we 
have $\lambda_{w(S)} \geqslant \lambda_{s(T)}$ or $\lambda_{w(T)} \geqslant \lambda_{s(S)}$, so $x_{\ell}$ satisfies coalitional assortativity.

In the procedure, $x_{\ell}$ can only contain a coalition of size larger than $\rho$ if for some $k=$ $1, \ldots, \ell$, it holds in Step (ii) of the procedure that $f_{T}\left(x_{k-1}\right)=\varnothing$. Since there is $i \in S^{k} \cap T$ such that $r_{i}\left(S^{k}\right) \geqslant r_{i}(T)$, it holds that $\bar{u}\left(S^{k}\right) \leqslant \bar{u}(T)$. If $S^{k}$ contains more than $\rho$ individuals and there is $i, j \in S^{k}$ such that $\lambda_{i} \neq \lambda_{j}$, then $\bar{u}\left(S^{k}\right)>\bar{u}\left(S^{k} \backslash\left\{s\left(S^{k}\right)\right\}\right) \geqslant \bar{u}(T)$, leading to a contradiction. Consequently, $x_{\ell}$ satisfies homophily.

If $Z\left(x_{\ell}\right) \neq \varnothing$, then $\left|Z\left(x_{\ell}\right)\right|<\rho$, as otherwise the procedure does not stop. By construction, it holds that for every $i \in \cup_{k=1}^{\ell} S^{k}$, for every $j \in N \backslash \cup_{k=1}^{\ell} S^{k}=Z\left(x_{\ell}\right)$ that $\lambda_{i} \leqslant \lambda_{j}$, so $x_{\ell}$ satisfies exclusion of the strong.

\section{A.4 Proof of Theorem 5.4}

The following lemma is helpful in the proof of Theorem 5.4.

Lemma A.2. Let $\Gamma=\left(N, X, E,\left\{\geq_{i}\right\}_{i \in N}\right)$ be a social environment induced by a competitive society $(N, \lambda, \rho)$. Let $x \in X$ and $S \in x$ be such that $|S|>\rho$ and $\lambda_{i} \neq \lambda_{j}$ for some $i, j \in S$. Then $f_{S \backslash\{w(S)\}}(x) \neq \varnothing$.

Proof. Consider the coalition $T=S \backslash\{w(S)\}$. Since $|T| \geqslant \rho$, in order to prove that $f_{S \backslash\{w(S)\}} \neq$ $\varnothing$, it suffices to show that $m_{i}(T)>m_{i}(S)$ for all $i \in T$. Observe that $\lambda_{w(S)}<\sum_{i \in S} \lambda_{i} /|S|$ and thus $\bar{u}(T)>\bar{u}(S)$. It follows that, for every $i \in T, m_{i}(T)=\bar{u}(T)>\bar{u}(S)=m_{i}(S)$.

Proof of Theorem 5.4. The proof consists of three parts. We show first that $\bar{X} \subseteq C$, next that $\bar{X} \supseteq C$, thereby showing that the Core coincides with the set of top-down segregated states, and finally that the Myopic Stable Set coincides with the Core.

Part 1. $\bar{X} \subseteq C$.

Let $\bar{x} \in X$ be a top-down segregated state, where $\bar{x}=\left\{S^{1}, \ldots, S^{m}\right\}, \mathcal{P}(\bar{x})=\left\{S^{1}, \ldots, S^{\ell}\right\}$, and, for every $k \in\{1, \ldots, \ell-1\}, \lambda_{s\left(S^{k}\right)} \leqslant \lambda_{w\left(S^{k+1}\right)}$. Moreover, we write $S^{1}=\left\{s_{1}^{1}, \ldots, s_{p}^{1}\right\}$ with $s_{1}^{1}<\cdots<s_{p}^{1}$.

Towards a contradiction, suppose that $\bar{x} \notin C$. Thus, there exists a coalition $T=$ $\left\{t_{1}, \ldots, t_{q}\right\}$ with $t_{1}<\cdots<t_{q}$ and a state $y$ such that $y \in f_{T}(\bar{x})$. Notice that any deviating coalition has to be productive, i.e., $|T| \geqslant \rho$. We proceed by induction.

Step 1: $S^{1} \cap T=\varnothing$.

Notice that coalition $S^{1}$ is productive. We consider two cases: a case in which, for all $i, j \in S^{1}, \lambda_{i}=\lambda_{j}$ and a case in which there are some $i, j \in S^{1}$ with $\lambda_{i} \neq \lambda_{j}$.

In the former case, we have that $\bar{u}\left(S^{1}\right)=\lambda_{1} \geqslant \bar{u}(T)$ as, by assumption, 1 is a strong individual in the society. Therefore, it holds that $S^{1} \cap T=\varnothing$. 
In the latter case, homophily of $\bar{x}$ implies that $\left|S^{1}\right|=\rho$. We define the collection of $\rho$ strongest individuals in $T$ by $T^{\prime}=\left\{t_{1}, \ldots, t_{\rho}\right\}$. It holds that $\bar{u}\left(T^{\prime}\right) \geqslant \bar{u}(T)$. For $i=1, \ldots, \rho$, it holds that $\lambda_{s_{i}} \geqslant \lambda_{t_{i}}$, so we have that $\bar{u}\left(S^{1}\right) \geqslant \bar{u}\left(T^{\prime}\right) \geqslant \bar{u}(T)$, and it follows that $S^{1} \cap T=\varnothing$.

Step 2: If $\bigcup_{\kappa=1}^{k} S^{\kappa} \cap T=\varnothing$, then $S^{k+1} \cap T=\varnothing$.

If $k \geqslant \ell$, then $S^{k+1} \notin \mathcal{P}(\bar{x})$. As $\bigcup_{\kappa=1}^{k} S^{\kappa} \cap T=\varnothing$ and $|Z(\bar{x})|<\rho$ it follows that $|T|<\rho$, a contradiction to $|T| \geqslant \rho$.

If $k<\ell$, then $S^{k+1} \in \mathcal{P}(\bar{x})$ and we write $S^{k+1}=\left\{s_{1}^{k+1}, \ldots, s_{p}^{k+1}\right\}$ with $s_{1}^{k+1}<\cdots<s_{p}^{k+1}$. If for all $i, j \in S^{k+1}, \lambda_{i}=\lambda_{j}$, then $\bar{u}\left(S^{k+1}\right)=\lambda_{s_{1}^{k+1}} \geqslant \bar{u}(T)$ as, by assumption, $s_{1}^{k+1}$ is a strong individual in $N \backslash \bigcup_{\kappa=1}^{k} S^{\kappa}$. Therefore, for all $i \in S^{k+1} \cap T$, it holds that $m_{i}\left(S^{k+1}\right) \geqslant m_{i}(T)$, so $S^{k+1} \cap T=\varnothing$. If there is some $i, j \in S^{k+1}$ with $\lambda_{i} \neq \lambda_{j}$, by homophily, $\left|S^{k+1}\right|=\rho$. We define $T^{\prime}=\left\{t_{1}, \ldots, t_{\rho}\right\}$, so $\bar{u}\left(T^{\prime}\right) \geqslant \bar{u}(T)$. Since $\lambda_{s_{i}^{k+1}} \geqslant \lambda_{t_{i}}$ for $i=1, \ldots, \rho$, we have that $\bar{u}\left(S^{k+1}\right) \geqslant \bar{u}\left(T^{\prime}\right) \geqslant \bar{u}(T)$. It follows that $S^{k+1} \cap T=\varnothing$.

By Steps 1 and 2 it follows that $T=\varnothing$ and we have obtained a contradiction. Consequently, it holds that $\bar{x} \in C$.

Part 2. $\bar{X} \supseteq C$. Let some $y \in C$ be given. Towards a contradiction, suppose that $y$ is not top-down segregated.

If homophily is violated, then there exists a coalition $S \in y$ with $|S|>\rho$ such that for some $i, j \in S, \lambda_{i}>\lambda_{j}$. Thus, by Lemma A.2, it holds that $f_{S \backslash\{w(S)\}}(y) \neq \varnothing$, a contradiction to $y$ being in the Core.

If coalitional assortativity is violated, then there are coalitions $S, T \in \mathcal{P}(y)$ such that $\lambda_{w(S)}<\lambda_{s(T)}$ and $\lambda_{w(T)}<\lambda_{s(S)}$. We write $S=\left\{s_{1}, \ldots, s_{p}\right\}$ and $T=\left\{t_{1}, \ldots, t_{q}\right\}$ with $s_{1}<\cdots<s_{p}$ and $t_{1}<\cdots<t_{q}$. We show that for $S^{\prime}=\left\{s_{1}, \ldots, s_{p-1}, t_{1}\right\}$ it holds that $f_{S^{\prime}}(y) \neq \varnothing$ or for $T^{\prime}=\left\{t_{1}, \ldots, t_{q-1}, s_{1}\right\}$ it holds that $f_{T^{\prime}}(y) \neq \varnothing$.

Since $|S|=\left|S^{\prime}\right| \geqslant \rho$ and $\lambda_{q_{1}}>\lambda_{s_{p}}$, we have, for all $i \in S \cap S^{\prime}=S^{\prime} \backslash\left\{t_{1}\right\}$,

$$
m_{i}(S)=\bar{u}(S)=\frac{\lambda_{s_{p}}+\sum_{i=1}^{p-1} \lambda_{s_{i}}}{|S|}<\frac{\lambda_{y_{1}}+\sum_{i=1}^{p-1} \lambda_{s_{i}}}{\left|S^{\prime}\right|}=\bar{u}\left(S^{\prime}\right)=m_{i}\left(S^{\prime}\right) .
$$

By symmetry, it also holds for all $i \in T \cap T^{\prime}=T^{\prime} \backslash\left\{s_{1}\right\}$ that $m_{i}(T)<m_{i}\left(T^{\prime}\right)$.

Towards a contradiction, suppose that $s_{1}=s(S)$ has no incentive to participate in $T^{\prime}$ and $t_{1}=s(T)$ has no incentive to form $S^{\prime}$, so $\bar{u}(S) \geqslant \bar{u}\left(T^{\prime}\right)$ and $\bar{u}(T) \geqslant \bar{u}\left(S^{\prime}\right)$. Thus, by definition,

$$
\begin{aligned}
& \frac{\sum_{i \in S} \lambda_{i}}{p} \geqslant \frac{\lambda_{S(S)}}{q}+\frac{\sum_{i \in T \backslash\{w(T)\}} \lambda_{i}}{q}, \\
& \frac{\sum_{i \in T} \lambda_{i}}{q} \geqslant \frac{\lambda_{S(T)}}{p}+\frac{\sum_{i \in S \backslash\{w(S)\}} \lambda_{i}}{p} .
\end{aligned}
$$


These expressions can be simplified to get

$$
\begin{aligned}
& q \sum_{i \in S} \lambda_{i} \geqslant p \lambda_{s(S)}+p \sum_{i \in T \backslash\{w(T)\}} \lambda_{i}, \\
& p \sum_{i \in T} \lambda_{i} \geqslant q \lambda_{s(T)}+q \sum_{i \in S \backslash\{w(S)\}} \lambda_{i} .
\end{aligned}
$$

Adding up these two inequalities and simplifying results in

$$
q \lambda_{w(S)}+p \lambda_{w(T)} \geqslant p \lambda_{s(S)}+q \lambda_{s(T)}
$$

leading to a contradiction since $\lambda_{w(S)}<\lambda_{s(T)}$ and $\lambda_{w(T)}<\lambda_{s(S)}$. Consequently, $s_{1}$ is strictly better off participating in $T^{\prime}$ or $t_{1}$ is strictly better off forming $S^{\prime}$.

We have shown that $f_{S^{\prime}}(y) \neq \varnothing$ or $f_{T^{\prime}}(y) \neq \varnothing$, contradicting that $y \in C$. Consequently, $y$ satisfies coalitional assortativity.

Suppose $y$ violates exclusion of the weak. If $|Z(y)| \geqslant \rho$, then we have $f_{Z(y)}(y) \neq \varnothing$, a contradiction. It remains to consider the case $|Z(y)|<\rho$. There exists $j \in Z(y), S \in \mathcal{P}(y)$, and $i \in S$ such that $\lambda_{i}<\lambda_{j}$. We write $S=\left\{s_{1}, \ldots, s_{p}\right\}$ with $s_{1}<\cdots<s_{p}$. Consider the coalition $T=\left\{s_{1}, \ldots, s_{p-1}, j\right\}$. We argue that $f_{T}(y) \neq \varnothing$. Notice that $m_{j}(Z(y))=$ $0<m_{j}(T)$, so $j$ has an incentive to deviate. It remains to be shown that also members of $\left\{s_{1}, \ldots, s_{p-1}\right\}=S \cap T$ have an incentive to deviate. Since $|S|=|T|$, for all $i \in S \cap T$ the following inequality holds,

$$
m_{i}(S)=\bar{u}(S)=\frac{\lambda_{s_{p}}+\sum_{i=1}^{p-1} \lambda_{i}}{|S|}<\frac{\lambda_{j}+\sum_{i=1}^{p-1} \lambda_{i}}{|T|}=\bar{u}(T)=m_{i}(T) .
$$

We have obtained a contradiction with $y \in C$. Consequently, $y$ satisfies exclusion of the weak.

\section{Part 3. The Myopic Stable Set coincides with the Core.}

Notice that the Core trivially satisfies deterrence of external deviations. In order to show that it coincides with the Myopic Stable Set, we have to show that it satisfies iterated external stability. The minimality requirement follows from the fact that the Core is a subset of the Myopic Stable Set.

The proof of iterated external stability is constructive using the following procedure, which, given an initial state $x_{0} \in X \backslash C$, generates a finite sequence of productive coalitions $S^{1}, \ldots, S^{\ell}$ and states $x_{1}, \ldots, x_{\ell}$ such that, for $k=1, \ldots, \ell, x_{k} \in \tilde{f}_{S^{k}}\left(x_{k-1}\right)$ and $x_{\ell}$ is a topdown segregated state. The coalitions $S^{k}$ are all disjoint and chosen such that their members have the highest productivity among players in $N \backslash\left(\cup_{\kappa=1}^{k-1} S^{\kappa}\right)$.

Start with a state $x_{0} \in X \backslash C$. We define $k=1$.

(i) If $\left|N \backslash \bigcup_{\kappa=1}^{k-1} S^{\kappa}\right|<\rho$, then the procedure ends. Otherwise, move to (ii). 
(ii) Consider a coalition $T \subseteq N \backslash \bigcup_{\kappa=1}^{k-1} S^{\kappa}$ with $|T|=\rho$ such that for all $i \in T$ and for all $j \in N \backslash\left(\bigcup_{\kappa=1}^{k-1} S^{\kappa} \cup T\right)$ it holds that $\lambda_{i} \geqslant \lambda_{j}$. If $f_{T}\left(x_{k-1}\right) \neq \varnothing$, then define $S^{k}=T$. If $f_{T}\left(x_{k-1}\right)=\varnothing$, then there is $i \in T$ who belongs to a productive coalition $S \in x_{k-1}$ such that $m_{i}(S) \geqslant m_{i}(T)$. For all $i \in S$, for all $j \in N \backslash\left(\bigcup_{\kappa=1}^{k-1} S^{\kappa} \cup S\right)$, it holds that $\lambda_{i} \geqslant \lambda_{j}$. In this case, define $S^{k}=S$. Define $x_{k}=y\left(x_{k-1}, S^{k}\right)$. Increase $k$ by 1 and move back to (i).

The iteration takes $\ell \leqslant n / \rho$ steps and finally generates the state $x_{\ell}$. We have that $\mathcal{P}\left(x_{\ell}\right)=\left\{S^{k} \mid k \in\{1, \ldots, \ell\}\right.$. It holds that $x_{\ell} \in \tilde{f}^{\ell}\left(x_{0}\right)$ since, for every $k \in\{1, \ldots, \ell\}$, $x_{k} \in f_{S^{k}}\left(x_{k-1}\right)$ or $x_{k}=x_{k-1}$. It remains to be shown that $x_{\ell}$ belongs to the Core. By Parts 1 and 2 of the proof, we need to show that $x_{\ell}$ is top-down segregated.

We have that $\mathcal{P}\left(x_{\ell}\right)=\left\{S^{k} \mid k \in\{1, \ldots, \ell\}\right.$. By construction it holds that $\lambda_{w\left(S^{1}\right)} \geqslant$ $\lambda_{s\left(S^{2}\right)} \geqslant \cdots \geqslant \lambda_{w\left(S^{\ell-1}\right)} \geqslant \lambda_{s\left(S^{\ell}\right)}$. It directly follows that for all $S, T \in \mathcal{P}\left(x_{\ell}\right)$ with $S \neq T$, we have $\lambda_{w(S)} \geqslant \lambda_{s(T)}$ or $\lambda_{w(T)} \geqslant \lambda_{s(S)}$, so $x_{\ell}$ satisfies coalitional assortativity.

In the procedure, $x_{\ell}$ can only contain a coalition of size larger than $\rho$ if for some $k=$ $1, \ldots, \ell$, it holds in Step (ii) of the procedure that $f_{T}\left(x_{k-1}\right)=\varnothing$. Since there is $i \in S^{k} \cap T$ such that $m_{i}\left(S^{k}\right) \geqslant m_{i}(T)$, it holds that $\bar{u}\left(S^{k}\right) \geqslant \bar{u}(T)$. If $S^{k}$ contains more than $\rho$ individuals and there is $i, j \in S^{k}$ such that $\lambda_{i} \neq \lambda_{j}$, then $\bar{u}\left(S^{k}\right)<\bar{u}\left(S^{k} \backslash\left\{w\left(S^{k}\right)\right\}\right) \leqslant \bar{u}(T)$, leading to a contradiction. Consequently, $x_{\ell}$ satisfies homophily.

If $Z\left(x_{\ell}\right) \neq \varnothing$, then $\left|Z\left(x_{\ell}\right)\right|<\rho$, as otherwise the procedure does not stop. By construction, it holds that for every $i \in \cup_{k=1}^{\ell} S^{k}$, for every $j \in N \backslash \cup_{k=1}^{\ell} S^{k}=Z\left(x_{\ell}\right)$ that $\lambda_{i} \geqslant \lambda_{j}$, so $x_{\ell}$ satisfies exclusion of the weak.

\section{References}

Acemoglu, D., G. Egorov, and K. Sonin (2008), "Coalition Formation in Non-Democracies", Review of Economic Studies, 75, 987-1009.

Banerjee, S., H. Konishi, And T. Sönmez (2001), "Core in a Simple Coalition Formation Game," Social Choice and Welfare, 18, 135-153.

BarberÀ, S., C. Beviá, And C. Ponsatí (2015), "Meritocracy, Egalitarianism and the Stability of Majoritarian Organizations," Games and Economic Behavior, 91, 237-257.

Beviá, C., L. Córchon, and A. Romero-Medina (2017), "Relinquishing Power, Exploitation and Political Unemployment in Democratic Organizations," Social Choice and Welfare, 49, 735-753.

Bogomolnaia, A., And M.O. JaCKsOn (2002), "The Stability of Hedonic Coalition Structures," Games and Economic Behavior, 38, 201-230. 
Bracha, A., U. Gneezy, And G. Loewenstein (2015), "Relative Pay and Labor Supply," Journal of Labor Economics, 33, 297-315.

Card, D., A. Mas, E. Moretti, And E. Saez (2012), "Inequality at Work: The Effect of Peer Salaries on Job Satisfaction," American Economic Review, 102, 2981-3003.

Clark, A.E., P. Frijters, and M.A. Shields (2008), "Relative Income, Happiness, and Utility: An Explanation for the Easterlin Paradox and Other Puzzles," Journal of Economic Literature, 46, 95-144.

Demuynck, T., P.J.J. Herings, R.D. Saulle, and C. Seel (2018), "The Myopic Stable Set for Social Environments," forthcoming in Econometrica.

Duesenberry, J.S. (1949), Income, Saving, and the Theory of Consumer Behavior, Harvard University Press, Cambridge.

Dufwenberg, M., P. Heidhues, G. Kirchsteiger, F. Riedel, And J. Sobel (2011), "Other-regarding Preferences in General Equilibrium," Review of Economic Studies, 78, 613-639.

Ferrer-I-Carbonell, A. (2005), "Income and Well-being: An Empirical Analysis of the Comparison Income Effect," Journal of Public Economics, 89, 997-1019.

Frank, R.H. (1985), Choosing the Right Pond: Human Behavior and the Quest for Status, Oxford University Press, New York.

Goyal, S., P. Hernández, G. Martínez-Cánovas, F. Moisan, M. Muñoz-Herrera, AND A. SÁnchez (2018), "Integration and Diversity," Working Paper, 1-56.

Hafer, C. (2006), "On the Origins of Property Rights: Conflict and Production in the State of Nature," Review of Economic Studies, 73, 119-143.

Hart, S., And M. Kurz (1983), "Endogenous Formation of Coalitions," Econometrica, $51,1047-1064$.

Luttmer, E.F.P. (2005), "Neighbors as Negatives: Relative Earnings and Well-being," Quarterly Journal of Economics, 120, 963-1002.

Maccheroni, F., M. Marinacci, And A. Rustichini (2012), "Social Decision Theory: Choosing within and between Groups," Review of Economic Studies, 79, 1591-1636.

Milchtaich, I., And E. Winter (2002), "Stability and Segregation in Group Formation," Games and Economic Behavior, 38, 318-346. 
Morelli, M., And I.-U. PARK (2016), "Internal Hierarchy and Stable Coalition Structures," Games and Economic Behavior, 96, 90-96.

Muthoo, A. (2004), "A Model of the Origins of Basic Property Rights," Games and Economic Behavior, 49, 288-312.

Piccione, M., And R. Razin (2009), "Coalition Formation under Power Relations," Theoretical Economics, 4, 1-15.

Piccione, M., And A. Rubinstein (2007), "Equilibrium in the Jungle," Economic Journal, 117, 883-896.

RAY, D. (2007), A Game-theoretic Perspective on Coalition Structures, Oxford University Press, Oxford.

Ray, D., And R. Vohra (2014), "Coalition Formation," in H.P. Young and S. Zamir (eds.), Handbook of Game Theory, Volume 4, Elsevier, Amsterdam, pp. 239-326.

WAtтs, A. (2007), "Formation of Segregated and Integrated Groups," International Journal of Game Theory, 35, 505-519. 